\title{
Effect of Co-initiator on the Size Distribution of the Stable Poly(Styrene-co- Divinylbenzene) Microspheres in Acetone/Water Mixture
}

\author{
Jin Young Choi, Kangseok Lee, Byung Hyung Lee, and Soonja Choe* \\ Department of Chemical Engineering, Inha University, Incheon 402-751, Korea
}

Received September 23, 2008; Revised December 2, 2008; Accepted December 3, 2008

\begin{abstract}
Stable poly(styrene-co-divinylbenzene) [P(St-co-DVB)] microspheres with narrow size distribution were synthesized in the presence of 2,2'-azobis(2,4-dimethyl valeronitrile) (V-65) and co-initiator in an acetone/water mixture in the precipitation polymerization at $53{ }^{\circ} \mathrm{C}$ for $24 \mathrm{~h}$. Potassium peroxodisulfate (KPS), ammonium peroxodisulfate (APS) and sodium peroxodisulfate (NaPS) were used as co-initiators. The optimum ratio of acetone to water for the formation of a narrow distribution of $\mathrm{P}(\mathrm{St}-\mathrm{co}$-DVB) particles was 49:11 (g/g). The optimum co-initiator compositions for narrow distribution were 9:1 (g/g) for V-65 to KPS, 11:1 for V-65 to APS and 6:1 for V-65 to NaPS. The yield for these compositions was $54 \sim 57 \%$ and the largest particle size was obtained with the lowest zeta-potential and CV values. From the XPS measurements, the charge density was increased but the zeta potential decreased with increasing sulfur content, implying that the sulfate group provides the electrostatic stabilization on the particle surface. This suggested that the self-crosslinking between styrene and DVB, the electrostatic stabilization of initiators, and the balanced hydrophobic and hydrophilic properties of the solvents are responsible for the formation of stable $\mathrm{P}(\mathrm{St}-\mathrm{co}$-DVB) spherical particles with narrow size distribution.
\end{abstract}

Keywords: poly(styrene-co-divinylbenzene), precipitation polymerization, co-initiator, co-solvent.

\section{Introduction}

In many fields of academic as well as industrial area, highly crosslinked micron-sized spherical polymer particles are widely studied because of their superior strength, thermal and solvent resistance, and anti-slip properties. ${ }^{1}$ These unique strengths enable them to be used to variety of applications such as surface coatings, printing and pharmaceutical industries, bio-medical devices, supporting phases for separation science, sensors, controlled release reservoirs and supports for metallic nano-colloids. ${ }^{2-6}$

In order to be applicable in the above area, the size control and narrow size distribution are the major concern. ${ }^{7}$ Various techniques including emulsion, dispersion and suspension polymerization were proposed to prepare the crosslinked submicron and micron-sized spherical particles. ${ }^{8-12}$ However, the preparation of crosslinked microspheres using the above conventional polymerizations has not been successful as the following. ${ }^{10-13}$ (1) Micron-sized polymer particles has not been produced in emulsion polymerization. (2) Highly crosslinked spherical particles have not been synthesized in dispersion polymerization, $0.5 \mathrm{wt} \%$ of crosslinking agent was the maximum. (3) Narrow distribution of the particle sizes has not been achieved in suspension polymerization.

*Corresponding Author. E-mail: sjchoe@inha.ac.kr
Instead, the precipitation polymerization has been used to prepare the highly crosslinked particles in micron-size. ${ }^{14} \mathrm{Up}$ today, the synthesis of highly crosslinked polymers using various self-crosslinkable monomers have widely been studied; poly(divinylbenzene) $[\mathrm{P}(\mathrm{DVB})]{ }^{15,16}$ poly(methacrylic acid-co-polyethylene oxide methyl ether methacrylate),${ }^{17}$ poly(methacrylate-co-DVB) ${ }^{18}$ poly(chloromethyl styrene-coDVB) ${ }^{19}$ poly(DVB-co-maleic anhydride), ${ }^{20}$ poly(methyl methacrylate- $c o-\mathrm{DVB}),{ }^{8,21,22}$ poly(styrene-co-DVB) $[\mathrm{P}(\mathrm{St}-\mathrm{co}-$ DVB) ${ }^{23}$ poly(acrylamide-co-DVB) ${ }^{24}$ and poly(glycidyl methacrylate- $c o-\mathrm{DVB}))^{25,26}$ In addition, mechanistic studies to elucidate the formation of stable microspheres in the absence of any stabilizer have been reported. During the precipitation polymerization, stabilization is achieved by a self-stabilizing mechanism via crosslinking of polymer chains. ${ }^{10}$ Polymer particles have some defects on uniform surface due to a density difference. ${ }^{18}$ Furthermore, acetonitrile is reported to be the only solvent for preparing crosslinked polymer particles in the precipitation polymerization. ${ }^{21,27}$

Much effort has paid to find other solvents instead of acetonitrile in the precipitation polymerization, which is one of expensive and environmentally unfriendly solvent. It was first reported that the stable $\mathrm{P}(\mathrm{St}-\mathrm{co}-\mathrm{DVB})$ spherical particles of $1.64 \sim 4.02 \mu \mathrm{m}$ in diameter were prepared using acetone/methanol mixture medium and V-65 as an initiator at $53{ }^{\circ} \mathrm{C}$ in the precipitation polymerization. ${ }^{28}$ The suitable sol- 
ubility parameter, polarity and viscosity of the solvent mixtures in the absence of acetonitrile were major concerns for the formation of spherical particles using co-solvent systems. ${ }^{28}$ However, the poor size distribution of the spherical particles was left for the further study. Recently, it was reported that the particle size distribution becomes narrower when the charge of the particle surface increases due to high electrostatic repulsion. ${ }^{29,30}$ In addition, it was proposed that the initiator efficiency would vary with the viscosity of solvent and that the rate coefficient for initiator decomposition could be affected by the environment polarity. ${ }^{31,32}$

The further study was extended to find other solvents system and enhance the uniformity of the crosslinked $\mathrm{P}(\mathrm{St}-\mathrm{co}$ DVB) $(50 / 50$ in molar ratio) spherical particles in the precipitation polymerization. Initiator, 2,2'-azobis(2,4-dimethyl valeronitrile) (V-65) and persulfate related co-initiators such as potassium peroxodisulfate (KPS), ammonium peroxodisulfate (APS) and sodium peroxodisulfate (NaPS) were able to prepare stable $\mathrm{P}(\mathrm{St}-\mathrm{co}$-DVB) microspheres with narrow size distribution in acetone/water mixture. In addition, the contribution of co-initiator along with an initiator, V-65, was verified for the formation of $\mathrm{P}(\mathrm{St}-\mathrm{co}-\mathrm{DVB})$ particles with narrow distribution.

\section{Experimental}

Materials and Polymerization. Styrene (Junsei Chemicals, Japan) and divinylbenzene (DVB: 55\% mixture of isomers, Aldrich, USA) were purified using an inhibitor removal column (Aldrich, USA) and stored at $-5{ }^{\circ} \mathrm{C}$ prior to use. Analytical grades of 2,2'-azobis(2,4-dimethyl valeronitrile) (V-65: Wako, Japan) as the main initiator, and potassium peroxodisulfate (KPS, Junsei Chemicals, Japan), ammonium peroxodisulfate (APS, Junsei Chemicals, Japan) and sodium peroxodisulfate (NaPS, Junsei Chemicals, Japan) were used as co-initiators without further purification. Analytical grade of acetone (Samchun, Korea) and double-distilled de-ionized water were used as the medium.

The polymerization ingredients simply consist of acetone, water, styrene, DVB, V-65 and co-initiators such as KPS, APS, or NaPS. The total amount of monomer mixture, styrene and DVB, was $4 \mathrm{wt} \%$ of the media and the compositions were 50 to 50 in molar ratio. The composition of acetone to water mixture was 49 to $11(\mathrm{~g} / \mathrm{g})$, respectively. The total concentration of V-65 and KPS was $2 \mathrm{wt} \%$ with respect to the monomer mixture and the co-initiator concentration was divided by the weight fraction. After charging polymerizing ingredients in a $100 \mathrm{~mL}$ one neck round bottom flask, nitrogen was purged for $15 \mathrm{~min}$ and sealed. Polymerization was carried out in an oil bath with an agitation speed of $30 \mathrm{rpm}$ at $53{ }^{\circ} \mathrm{C}$ for $24 \mathrm{~h}$. After completion of the polymerization, the resultant particles were collected by centrifugation and washed with methanol repeatedly.

Characterizations. The yield was calculated by gravi- metrically. A Scanning Electron Microscopy (SEM: Hitachi, S-4300, USA) was used to study the morphology of the synthesized P(St-co-DVB). The samples were prepared with a drop of diluted suspension on an aluminum tape and sputter-coated with platinum. The particle size and the coefficient of variation $(\mathrm{CV})$ were measured by particle size analyzer (LS230 ${ }^{\circledR}$, Beckman Coulter, USA). Total measurements were made at 6 scattering angles and three wavelengths, each at two polarization provided information about the differences between the particle sizes. The glass transition temperature of the sample was measured using the Differential Scanning Calorimeter (DSC: DSC-7, Perkin-Elmer, USA). The experimental procedure is described in elsewhere. ${ }^{8}$ Zeta-potential analyzer (ELS-Z, Photal Otsuka, Japan) was used to measure the charge of the surface particles. The dilute (slightly turbid) aqueous suspension of submicron particles was prepared by mixing 1 drop of the latex with DI water and pipette some of it into one of the square plastic cuvets which are supplied with the unit. The quantitative measure of the charge on colloidal particles in liquid suspension was calculated from its electrophoretic mobility.

\section{Results and Discussion}

Effect of Solvent Mixture and co-Initiators on the Formation of Spherical Particles with Narrow Size Distribution. Table I shows the reaction recipes employed in this experiment, where the variables are the composition difference in solvents and initiators. The Expt. 1 in Table I represents the recipe upon the variance of the solvent compositions to find the best fit inducing narrow size distribution at the fixed composition of initiator, 9:1 for V-65 to KPS, respectively.

Figure 1 is the SEM images of $\mathrm{P}(\mathrm{St}-\mathrm{co}-\mathrm{DVB})$ particles prepared by the recipe described in Expt. 1 by the precipitation polymerization at $53{ }^{\circ} \mathrm{C}$ for $24 \mathrm{~h}$. The limited compositions of solvents at 51:9 (Figure 1(a)) and 46:14 (Figure 1(f)) of acetone to water, respectively, induced unstable or coagulated $\mathrm{P}(\mathrm{St}-\mathrm{co}$-DVB) particles. However, the stable spherical particles were obtained between 50:10 and 47:13 of acetone to water ratio, respectively.

The measured values of particle diameter, $\mathrm{CV}$ and zeta potential of $\mathrm{P}(\mathrm{St}-\mathrm{co}$-DVB) particles from Figure 1 were plotted in Figure 2 and varied depending on the compositions of acetone to water. The largest diameter at $1.24 \mu \mathrm{m}$, the lowest CV value at $11.7 \%$ and the lowest zeta potential at $-3.49 \mathrm{eV}$ were observed at 49:11 of acetone to water, respectively. Thus, the best solvent composition of acetone to water for narrow size distribution is 49: 11. This result implies that the formation of stable $\mathrm{P}(\mathrm{St}-\mathrm{co}$-DVB) spherical particles with narrow size distribution is affected by the combination of hydrophobic and hydrophilic property of the solvents.

The yield varied between 54 and $57 \%$ for all compositions of V-65 to KPS with 49:11 of acetone to water, respec- 
Table I. Recipe Used in the Precipitation Polymerization of Styrene and Divinylbenzene in the Solvent Mixture of Acetone to Water in the Presence of Co-initiators

\begin{tabular}{|c|c|c|c|c|c|}
\hline \multirow{2}{*}{$\begin{array}{l}\text { Expt. } \\
\text { No. }\end{array}$} & \multirow{2}{*}{$\begin{array}{c}\text { Monomer } \\
\text { Styrene : } \\
\text { DVB }\end{array}$} & \multicolumn{2}{|c|}{ Initiator } & \multicolumn{2}{|c|}{ Medium } \\
\hline & & $\begin{array}{l}\text { Co-initia- } \\
\text { tor }\end{array}$ & $\begin{array}{c}\text { V-65:Co-I } \\
\text { (g/g) }\end{array}$ & $\begin{array}{l}\text { Acetone } \\
\text { (g) }\end{array}$ & $\begin{array}{l}\text { Water } \\
(\mathrm{g})\end{array}$ \\
\hline \multirow{6}{*}{$\begin{array}{c}\text { Solvent } \\
\text { Composition } \\
\text { (Expt. 1) }\end{array}$} & & \multirow{6}{*}{ KPS } & \multirow{6}{*}{$9: 1$} & 51 & 9 \\
\hline & & & & 50 & 10 \\
\hline & & & & 49 & 11 \\
\hline & & & & 48 & 12 \\
\hline & & & & 47 & 13 \\
\hline & & & & 46 & 14 \\
\hline \multirow{8}{*}{$\begin{array}{l}\text { Initiator } \\
\text { Composition } \\
\text { (Expt. 2) }\end{array}$} & & \multirow{8}{*}{ KPS } & $0: 100$ & \multirow{8}{*}{49} & \multirow{8}{*}{11} \\
\hline & & & $5: 1$ & & \\
\hline & & & $6: 1$ & & \\
\hline & & & $7: 1$ & & \\
\hline & & & $9: 1$ & & \\
\hline & & & $11: 1$ & & \\
\hline & & & $12: 1$ & & \\
\hline & & & $100: 0$ & & \\
\hline \multirow{7}{*}{$\begin{array}{l}\text { Initiator } \\
\text { Composition } \\
\text { (Expt. 3) }\end{array}$} & & \multirow{7}{*}{ APS } & $0: 100$ & \multirow{7}{*}{49} & \multirow{7}{*}{11} \\
\hline & & & $5: 1$ & & \\
\hline & & & $6: 1$ & & \\
\hline & & & $7: 1$ & & \\
\hline & & & $11: 1$ & & \\
\hline & & & $12: 1$ & & \\
\hline & & & $100: 0$ & & \\
\hline \multirow{7}{*}{$\begin{array}{l}\text { Initiator } \\
\text { Composition } \\
\text { (Expt. 4) }\end{array}$} & & \multirow{7}{*}{$\mathrm{NaPS}$} & $0: 100$ & \multirow{7}{*}{49} & \multirow{7}{*}{11} \\
\hline & & & $5: 1$ & & \\
\hline & & & $6: 1$ & & \\
\hline & & & $7: 1$ & & \\
\hline & & & $9: 1$ & & \\
\hline & & & $12: 1$ & & \\
\hline & & & $100: 0$ & & \\
\hline
\end{tabular}

tively. Since the 49: $11(\mathrm{~g} / \mathrm{g})$ of acetone to water gives the best results in the size distribution, this is selected for the further experiments. The Expt. 2 in Table I is carried out to set the best composition of initiators between V-65 and KPS from 5:1 to $12: 1$ including V-65 and KPS solely. The SEM photographs of the $\mathrm{P}(\mathrm{St}-\mathrm{co}-\mathrm{DVB})$ are shown in Figure 3. When KPS is solely used in Figure 3(a), no formation of spherical particles was observed due to the hydrophilic initiator, KPS. This can not be adequately soluble in the medium mixture consisting of large quantity of hydrophobic acetone
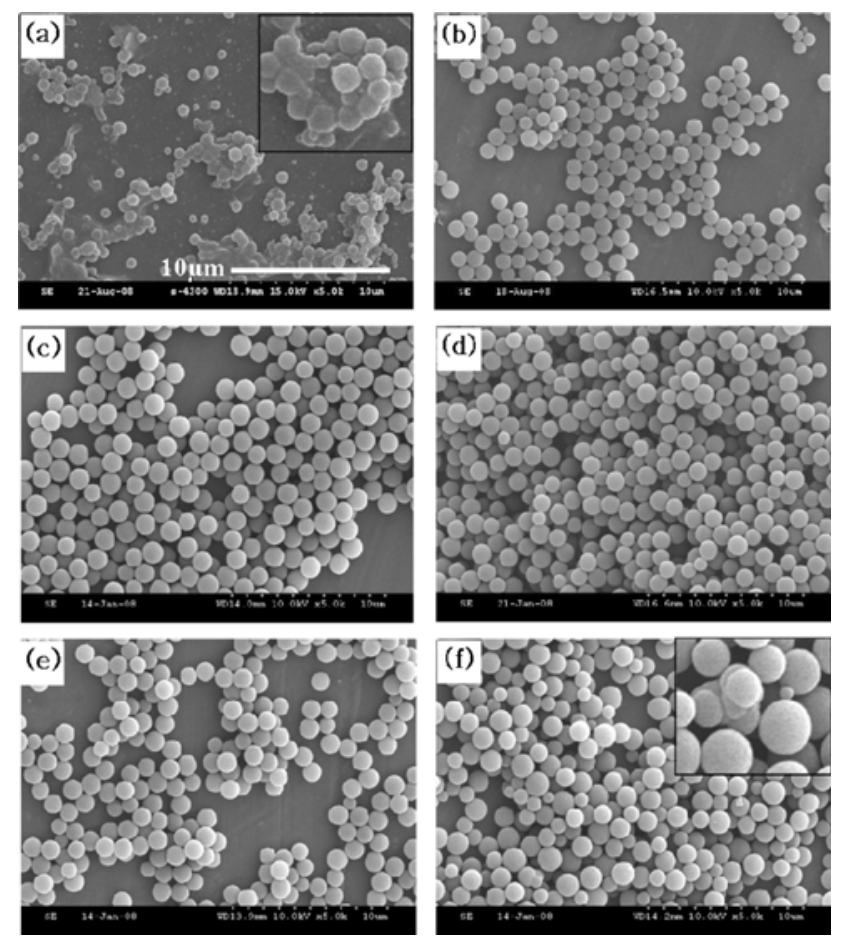

Figure 1. SEM photographs of $\mathrm{P}(\mathrm{St}-\mathrm{co}-\mathrm{DVB})$ microspheres prepared in various ratios of acetone to water (Expt. 1) by precipitation polymerization at $53{ }^{\circ} \mathrm{C}$ for $24 \mathrm{~h}$. The compositions of acetone to water (g/g) are (a) $51: 9$, (b) $50: 10$, (c) $49: 11$, (d) $48: 12$, (e) 47 $: 13$, (f) $46: 14$.

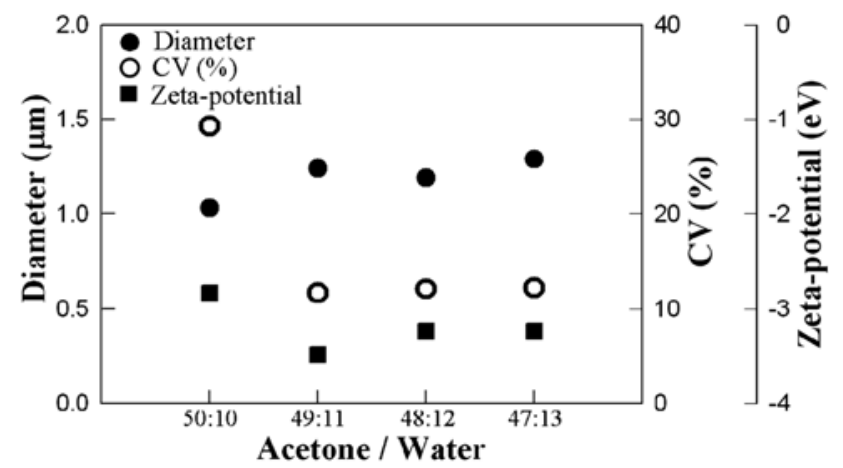

Figure 2. The particle diameter $(\mathbf{O}), \mathrm{CV}(\bigcirc)$ and zeta potential $(\boldsymbol{\square})$ of $\mathrm{P}(\mathrm{St}-\mathrm{co}-\mathrm{DVB})$ particles prepared with various ratios of acetone to water with $9: 1$ of $\mathrm{V}-65$ to KPS $(\mathrm{g} / \mathrm{g})$, respectively, at $53{ }^{\circ} \mathrm{C}$ for $24 \mathrm{~h}$ in the precipitation polymerization.

compared to hydrophilic water (49:11, respectively). On the other hand, when hydrophobic V-65 is solely used, coagulated particles are obtained as seen in Figure 3(h) due to the insufficient stability for the formation of particles. In addition, when the lack (5:1 of V-65 to KPS, respectively) or excess (12:1 of V-65 to KPS, respectively) of V-65 is used as seen in Figures 3(b) and 3(g), respectively, the partial coagulum is observed possibly due to the too large or too small quantity of hydrophilic initiator, KPS. On the other hand, when 

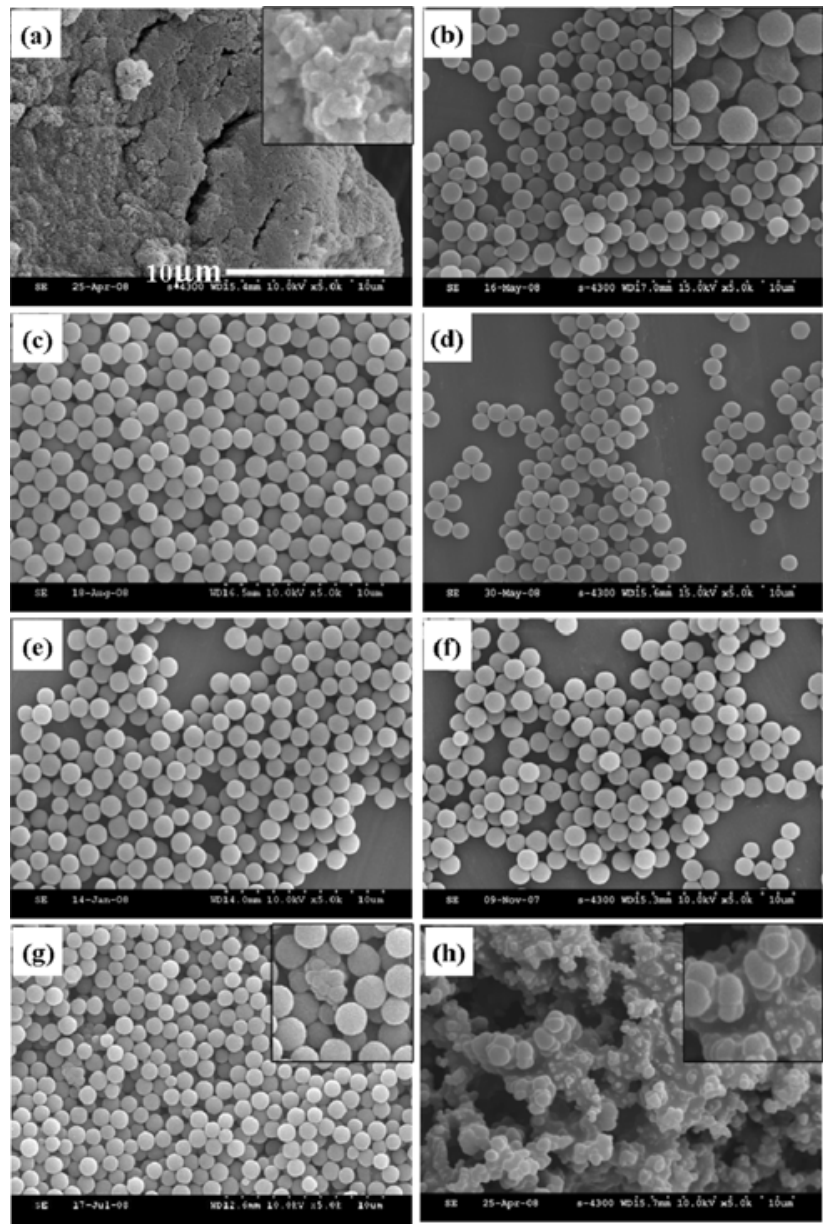

Figure 3. SEM photographs of $\mathrm{P}(\mathrm{St}-\mathrm{co}$-DVB) microspheres prepared with 49/11 (acetone/water) mixture and various ratios of initiators of V-65 to KPS in the precipitation polymerization at $53{ }^{\circ} \mathrm{C}$ for $24 \mathrm{~h}$ (Expt. 3). The compositions of V-65 to KPS (g/g) are (a) 0 : 100 , (b) $5: 1$, (c) $6: 1$, (d) $7: 1$, (e) $9: 1$, (f) $11: 1$, (g) $12: 1$, (h) 100:0.

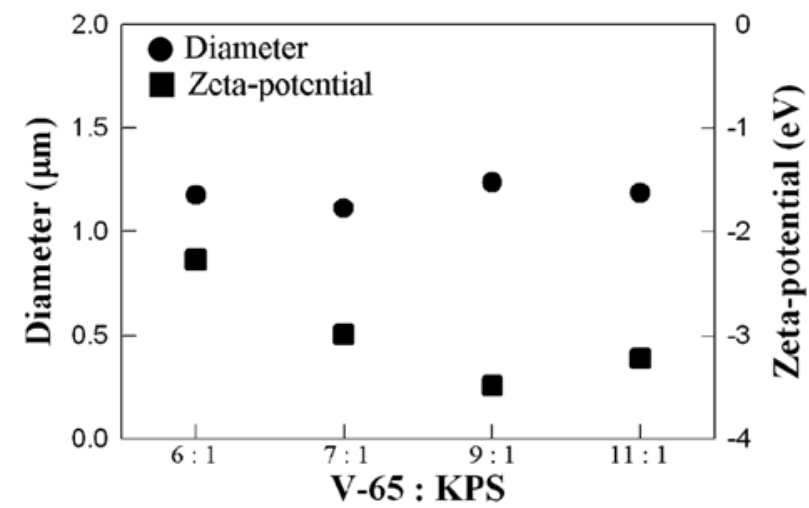

Figure 4. The particle diameter $(\boldsymbol{O})$ and zeta potential $(\boldsymbol{\square})$ of the spherical P(St-co-DVB) particles prepared with various compositions of V-65 to KPS (g/g).

suitable combination of initiators was used (the composition of V-65 to KPS is between 6: 1 and 11: 1, respectively), the stable spherical particles were obtained as seen in Figures
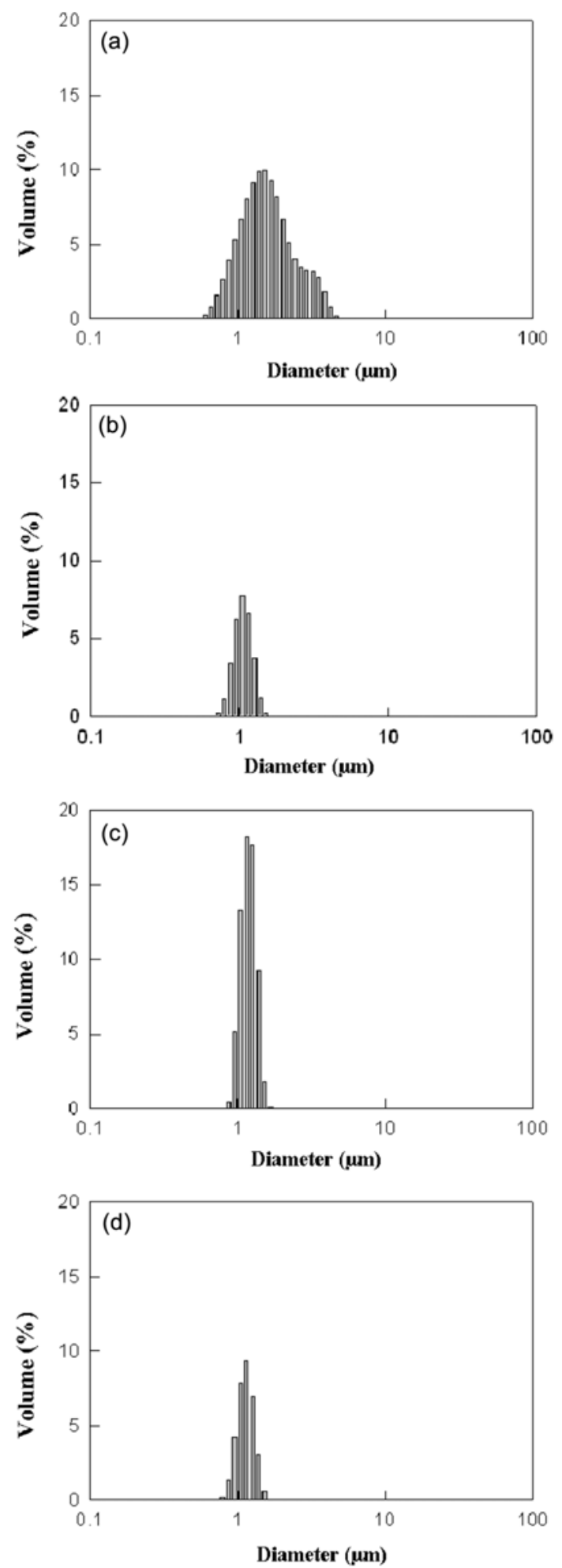

Figure 5. The size distribution of spherical $\mathrm{P}(\mathrm{St}-\mathrm{co}-\mathrm{DVB})$ particles prepared with various ratios of V-65: KPS. The compositions of V-65 to KPS (g/g) are (a) 6: 1, (b) 7: 1, (c) 9: 1, (d) 11: 1.

3(c) 3(f). Thus, the use of suitable amount of KPS as a coinitiator was necessary for the formation of $\mathrm{P}(\mathrm{St}-\mathrm{co}-\mathrm{DVB})$ 

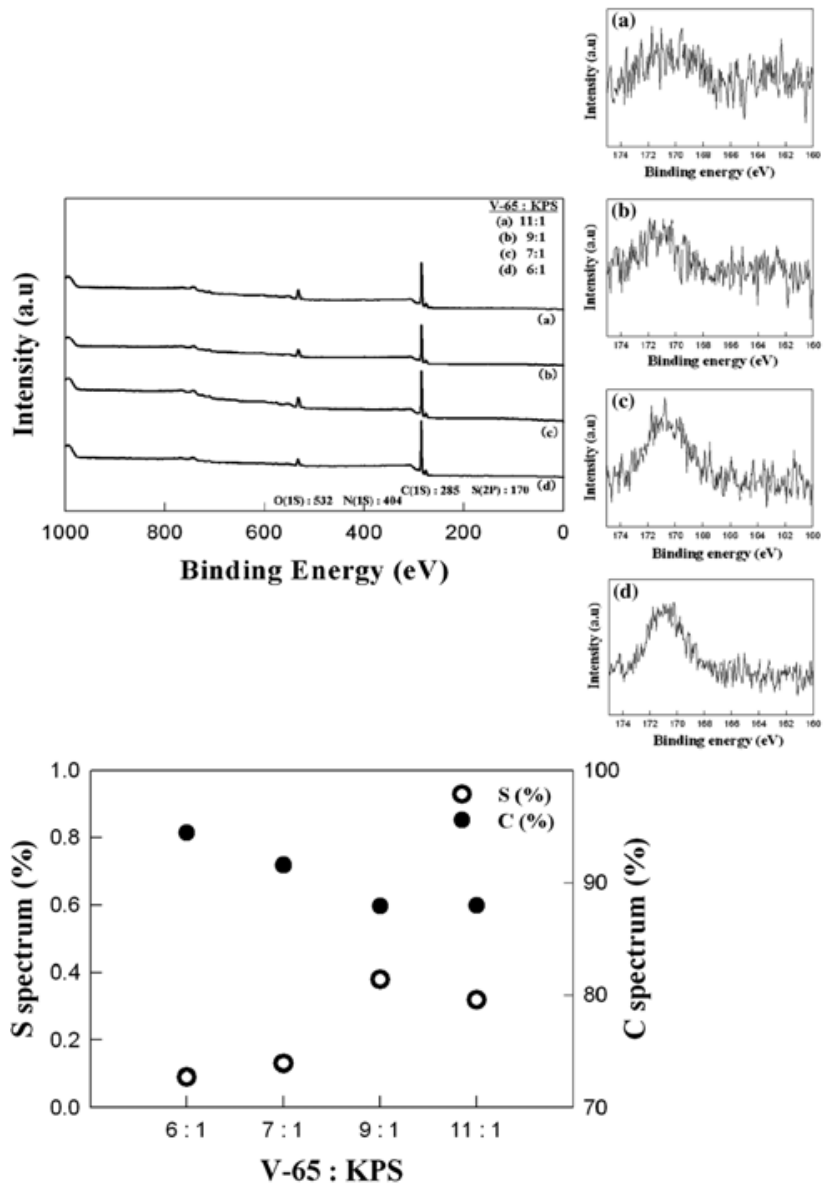

Figure 6. The relative sulfur and carbon contents of $\mathrm{P}(\mathrm{St}-\mathrm{co}$-DVB) particles using XPS measurement with various compositions of V-65: KPS (g/g); 6:1, 7:1, 9:1, and 11:1.

spherical particles with enhanced stability.

In order to obtain the best condition for the narrower size distribution, the measured particle diameter and zeta potential were plotted in Figure 4. The particle diameter varied from 1.11 to $1.24 \mu \mathrm{m}$ and the zeta potential varied from -2.27 to $-3.49 \mathrm{eV}$ for the $\mathrm{V}-65$ to KPS ratio between $6: 1$ and $11: 1$, respectively. In addition, the size distribution of the spherical $\mathrm{P}(\mathrm{St}-\mathrm{co}$-DVB) particles is drawn in Figure 5. The coefficient of variation (CV) representing the size distribution varied from 33.0 to $11.7 \%$. Thus, the narrower the size distribution, the higher the charge density (the lower the zeta potential) was obtained. This suggests that the narrow size distribution would be related to the large electrostatic stabilization induced from the charge density of co-initiator. ${ }^{31}$

In order to investigate the reason why the $9: 1$ ratio of $\mathrm{V}$ 65 to KPS is the narrowest distribution in particle sizes, the further experiment using XPS was carried out to verify the sulfate group in KPS. Since KPS sorely consists of sulfur, the content ratio of $\mathrm{C} 1 \mathrm{~s}$ at $285 \mathrm{eV}$ and $\mathrm{S} 2 \mathrm{p}$ at $170 \mathrm{eV}$ of binding energy for the surface $(10 \mathrm{~nm})$ of $\mathrm{P}(\mathrm{St}-\mathrm{co}-\mathrm{DVB})$ was measured and the result is plotted in Figure 6. Since the
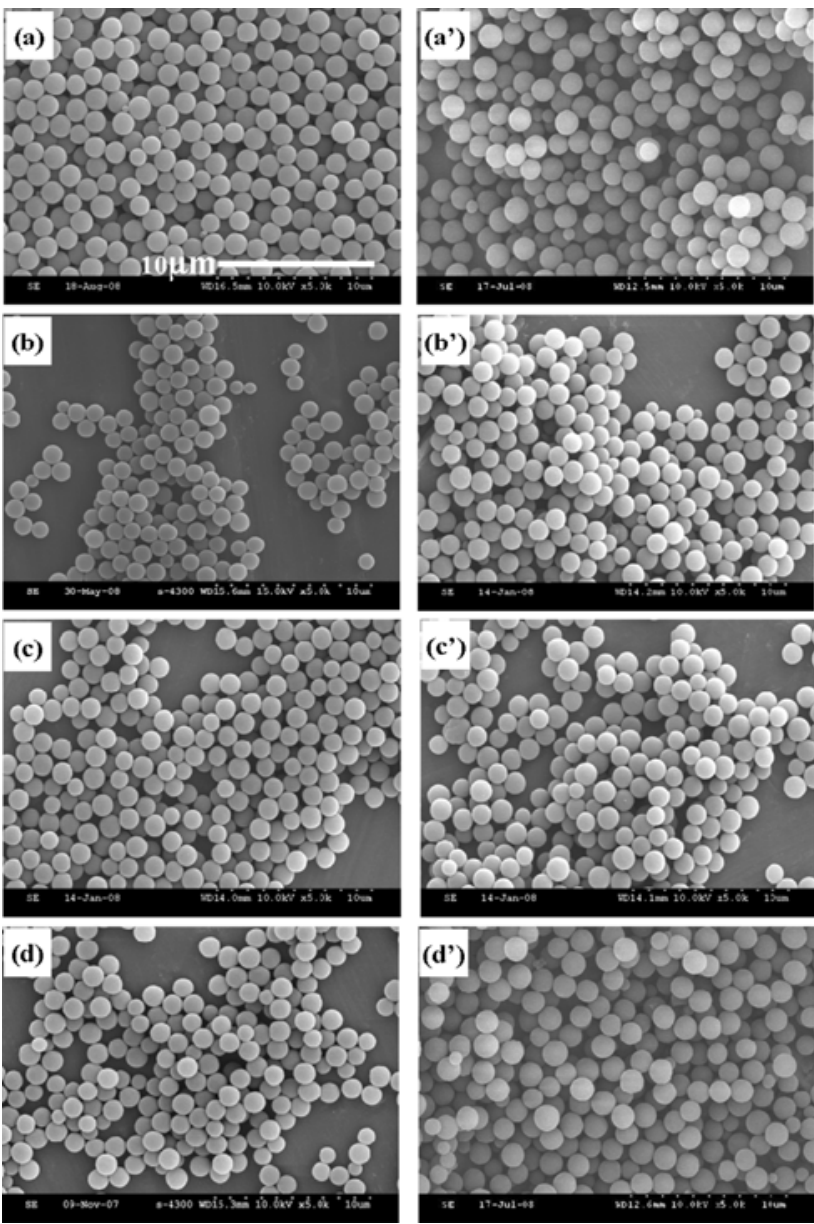

Figure 7. The SEM photographs of the $\mathrm{P}(\mathrm{St}-\mathrm{co}-\mathrm{DVB})$ particles before (a, b, c, d) and after swelling test (a', b', c'. d') by immersing them in THF for $24 \mathrm{~h}$. The initiator compositions of V-65 to KPS (g/g); (a, a') 6:1, (b, b') 7:1, (c, c') 9:1, (d, d') 11:1.

quantity of sulfur was very small, the enlarged peak was included in Figure 6. At 9:1 of V-65 to KPS ratio, the highest content of sulfur was obtained, implying that the higher the sulfur content, the higher the stable spherical $\mathrm{P}(\mathrm{St}-\mathrm{co}$ DVB) particles were obtained, which means the highest electrostatic stabilization with high content of electron charge.

From the above results from Figure 1 to 6 , the most monodisperse and stable spherical particles were obtained at 49:11 of acetone to water and 9:1 of V-65 to KPS ratio, respectively. This result implies that the formation of stable particles with narrow size distribution is affected not only by the balanced combination of hydrophobic and hydrophilic property of solvents, but also the electrostatic stabilization of initiators.

DSC analysis was performed using the particles prepared with various ratios of V-65 to KPS (from 6:1 to 11:1), but no glass transition temperature was obtained, implying that the $\mathrm{P}(\mathrm{St}-\mathrm{co}$-DVB) is highly crosslinked.

In order to verify the stability of the $\mathrm{P}(\mathrm{St}-\mathrm{co}-\mathrm{DVB})$ spher- 


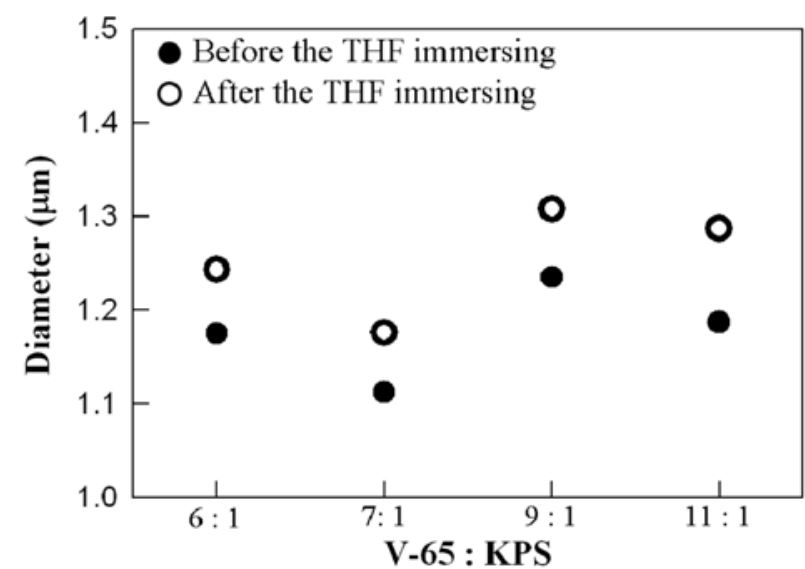

Figure 8. The variance of the diameter of the $\mathrm{P}(\mathrm{St}-\mathrm{co}-\mathrm{DVB})$ particles before $(O)$ and after $(\bigcirc)$ the swelling test by immersing them in THF for $24 \mathrm{~h}$ upon the compositions of V-65 to KPS $(\mathrm{g} / \mathrm{g})$ between $6: 1$ and 11:1.

ical particles, swelling test was carried out by immersing them in THF for $24 \mathrm{~h}$ and the results were drawn in Figure 7. No deformation or deterioration of the spherical particles was observed. The particle diameters before and after swelling were measured and the results were plotted in Figure 8. As seen in this figure, a slight increase between 3.4 and $5.1 \%$ in the particle diameter was obtained, thus the formed spherical particles were still stable even if the samples were immersed in organic solvents for a long time.

From the results shown in Figures 7 and 8 including DSC results, the $\mathrm{P}(\mathrm{St}-\mathrm{co}-\mathrm{DVB})$ spherical particles were highly crosslinked and solvent resistant due to the self-crosslinking between styrene and DVB, electrostatic stabilization of initiators and the balanced hydrophobic and hydrophilic properties of solvents.

Effect of Other Sulfate-type Initiators as Co-initiators. In order to verify the capability of electrostatic stabilization of water-soluble initiators, two other sulfate-derivative initiators such as APS and NaPS were also used. The Expt. 3 and Expt. 4 in Table I represent the recipe for the preparation of P(St-co-DVB) spherical particles using APS and NaPS, respectively. Figure 9 represents the SEM photographs of $\mathrm{P}(\mathrm{St}-\mathrm{co}$-DVB) microspheres prepared with 49:11 of acetone/water mixture and various compositions of initiators between V-65 and APS in the precipitation polymerization at $53{ }^{\circ} \mathrm{C}$ for $24 \mathrm{~h}$. When APS was solely used, no spherical particles were obtained, but very tiny nano-meter sized particles were formed as seen in Figure 9(a). The effect of APS on the present polymerization with co-solvents system seems to be the same as with KPS. In addition, when V-65 was solely used, coagulum was observed in Figure 9(h). When the composition of V-65: APS is 5: 1 and 12:1, the partial coagulum with unstable particles was formed as seen in Figures 9(b) and 9(g), respectively. On the other hand, when the composition of V-65 to APS is between 6:1 and
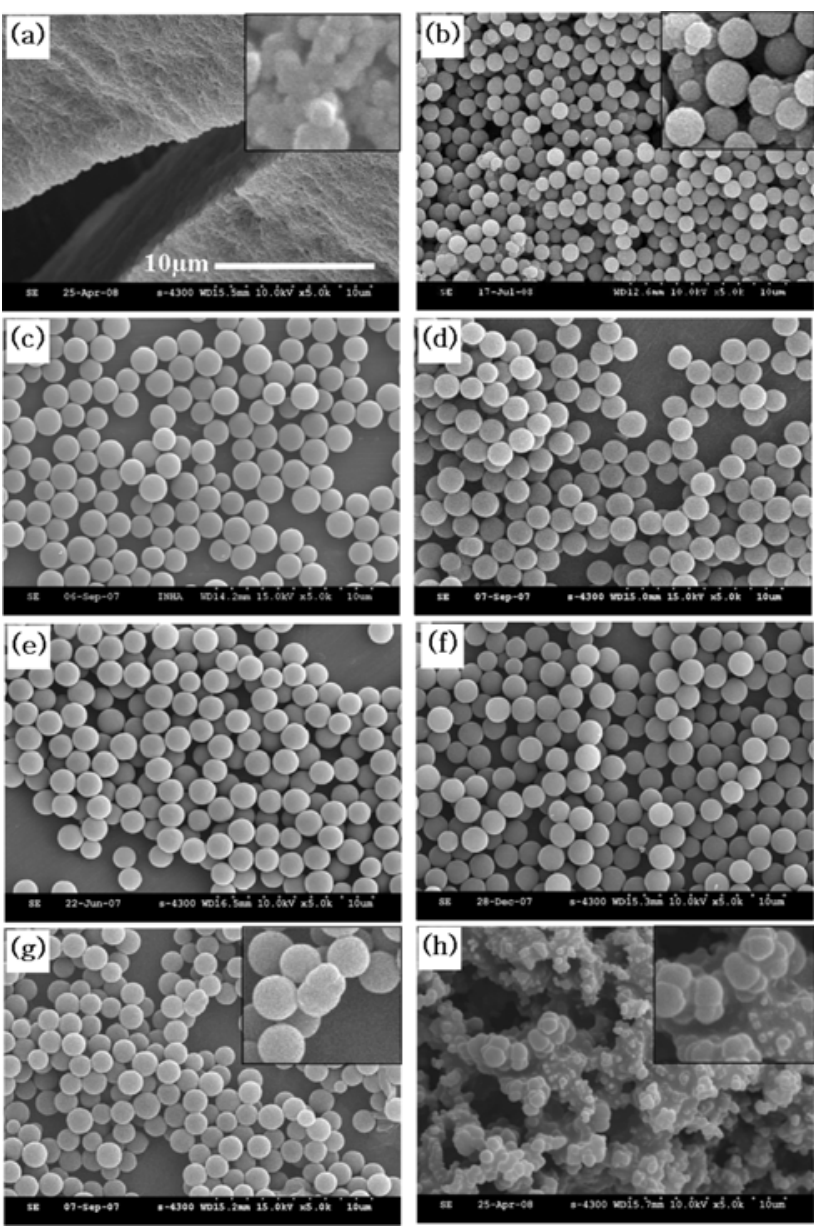

Figure 9. SEM photographs of $\mathrm{P}(\mathrm{St}-\mathrm{co}$-DVB) microspheres prepared with 49/11 (acetone/water) mixture and co-initiator, KPS withV-65 in the precipitation polymerization at $53{ }^{\circ} \mathrm{C}$ for $24 \mathrm{~h}$ (Expt. 3). The compositions of V-65 to APS are (g/g); (a) $0: 100$, (b) $5: 1$, (c) $6: 1$, (d) $7: 1$ (e) $9: 1$, (f) $11: 1$, (g) $12: 1$, (h) 100:0.

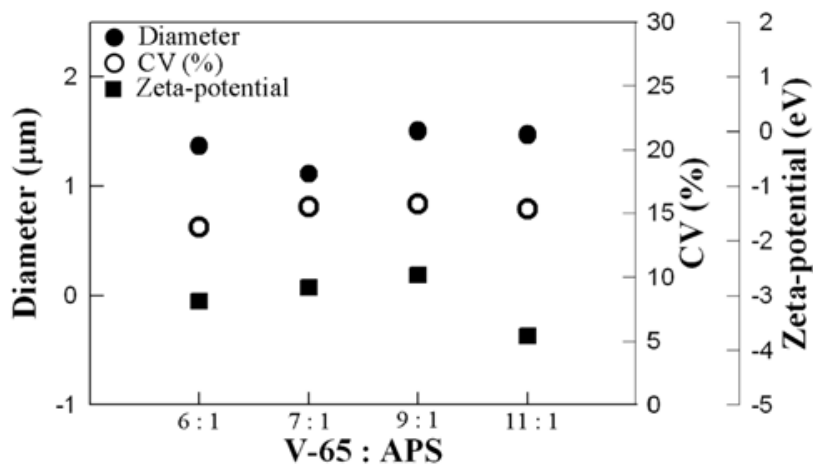

Figure 10. The particle diameter, $\mathrm{CV}$ values and zeta potential of the $\mathrm{P}(\mathrm{St}-\mathrm{co}$-DVB) spherical particles prepared with various compositions of V-65 to APS (g/g).

11: 1, the stable and relatively uniform particles are obtained in Figures 9(c) 9(f), respectively.

The measured particle diameter, CV and the zeta-poten- 

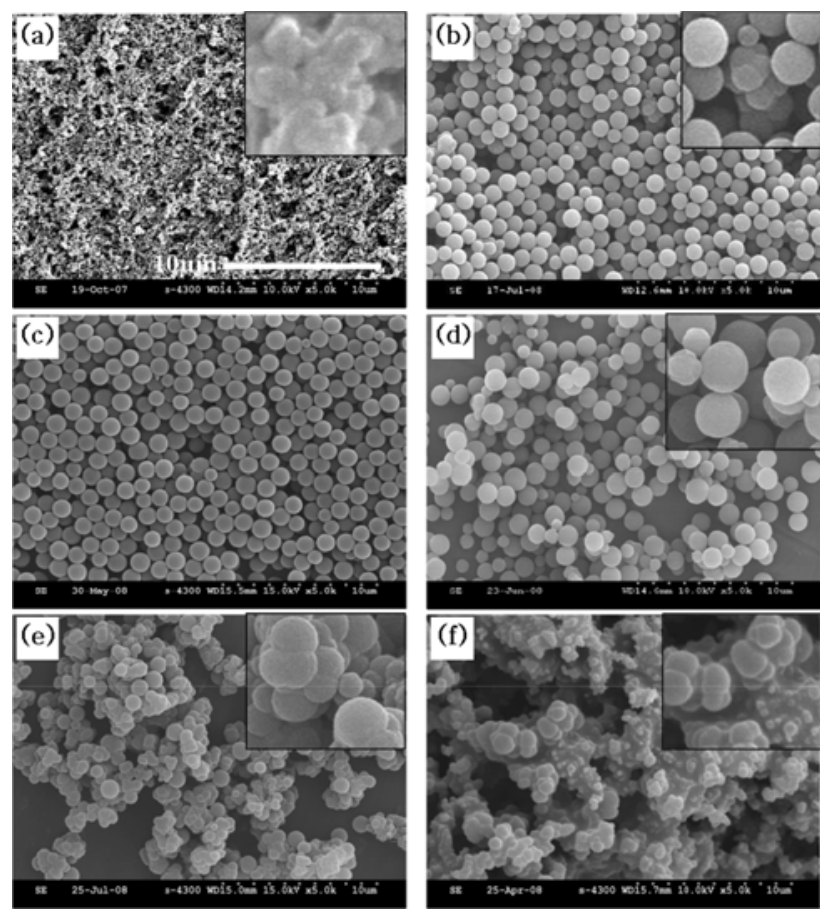

Figure 11. SEM photographs of $\mathrm{P}(\mathrm{St}-c o$-DVB) microspheres prepared with $49 / 11$ (acetone/water) mixtures and various ratios of initiators of V-65 to NaPS in the precipitation polymerization at $53{ }^{\circ} \mathrm{C}$ for $24 \mathrm{~h}$ (Expt. 4). The compositions of V-65 to NaPS are (g/g); (a) $0: 100$, (b) $5: 1$, (c) 6:1, (d) 7:1, (e) 9:1, (f) $100: 0$.

tial values from Figure 9 are plotted in Figure 10. The particles are micron-sized of $1.37,1.11,1.47$ and $1.50 \mu \mathrm{m}$, fairly narrow distribution with $\mathrm{CV}$ values of $13.9,15.5,15.7$ and 15.3 , and the zeta potentials with $-3.11,-2.85,-2.63$ and -3.73 for $6: 1,7: 1,9: 1$ and 11: 1 of V-65 to APS ratio, respectively. Within the experimental errors, all these four compositions are equally contributable. In overall, the best composition of V-65 to APS for narrow size distribution is 11:1 due to the largest diameter, and the lowest $\mathrm{CV}$ value and zeta potential.

In addition, NaPS is also used in the same recipe listed in Expt. 4 in Table I and the SEM photographs are drawn in Figure 11. Except the composition of V-65 to NaPS at $6: 1$ in Figure 11(c), no stable spherical particles of $\mathrm{P}(\mathrm{St}-\mathrm{co}-\mathrm{DVB})$ were obtained including NaPS sorely; the particle size of this is $1.13 \mu \mathrm{m}, \mathrm{CV}$ is $17.0 \%$ and the zeta potential is $-2.91 \mathrm{eV}$. It is still not verified yet, but it seems that the use of co-initiator over the specific ratio interferes with stabilization of particle. In addition, the cause of that phenomenon is not yet explicable, but we assume that the $\mathrm{K}^{+}, \mathrm{NH}^{+}, \mathrm{Na}^{+}$influence the formation of particles.

\section{Conclusions}

This study presents the novel technique to prepare stable $\mathrm{P}(\mathrm{St}-\mathrm{co}$-DVB) $(50 / 50$ in molar ratio) spherical particles with narrow size distribution in acetone/water mixture medium and V-65 as an initiator in the presence of co-initiators, KPS, APS and NaPS in the precipitation polymerization at $53{ }^{\circ} \mathrm{C}$ for $24 \mathrm{~h}$. The optimum composition of cosolvents, acetone to water, for narrow size distribution was 49:11(g/g), respectively. When V-65, KPS, APS or NaPS was solely used, no spherical particles were obtained. However, when co-initiators such as KPS, APS and NaPS consisting of persulfate group were used with V-65, the spherical P(Stco-DVB) particles with fairly narrow size distribution were obtained. The optimum compositions of co-initiators for narrow distribution were 9: $1(\mathrm{~g} / \mathrm{g})$ for V-65 to KPS, 11:1 for V-65 to APS and 6:1 for V-65 to NaPS, respectively. In all cases with these compositions, the yield varied between 54 and $57 \%$ for all compositions of V-65 to KPS in 49:11 of acetone to water, respectively. In addition, the largest particle size was obtained with the lowest zeta-potential and CV values. In the XPS measurement, the larger the charge density (the lower the zeta potential), the higher the sulfur content was obtained, implying that the sulfate group gives the stability of the spherical particle surface due to the electrostatic stabilization. In addition, from DSC thermograms and swelling test in THF, the $\mathrm{P}(\mathrm{St}-\mathrm{co}$-DVB) spherical particles were confirmed to be highly crosslinked and solvent resistant due to the self-crosslinking between styrene and DVB, electrostatic stabilization of initiators, and balanced hydrophobic and hydrophilic properties of solvents.

Aknowledgement. This work was financially supported by the Ministry of Education and Human Resources Development (MOE), the Ministry of Commerce, Industry and Energy (MOCIE), and the Ministry of Labor (MOLAB) through the fostering project of the Lab of Excellency during 2005-2007.

\section{References}

(1) M. Hattori, E. D. Sudol, and M. S. El-Aasser, J. Polym. Sci. Part A: Polym. Chem., 30, 2027 (1993).

(2) P. Bradna, P. Stern, O. Quadrat, and J. Snuparek, Colloid Polym. Sci., 273, 324 (1995).

(3) M. Okubo and T. Nakayama, Colloid Polym. Sci., 273, 530 (1994).

(4) G. Tepper and N. Levit, Ind. Eng. Chem. Res., 39, 4445 (2000).

(5) R. H. Mueller, C. Jacobs, and O. Kayser, Adv. Drug. Deliver. Rev., 47, 3 (2001).

(6) S. N. Li, X. L. Yang, and W. Q. Huang, Macromol. Chem. Phys., 206, 1967 (2005).

(7) G. Liu, X. Yang, and Y. Wang, Polym. Int., 56, 905 (2007).

(8) S. Yang, S. E. Shim, and S. Choe, J. Polym. Sci. Part A: Polym. Chem., 43, 1309 (2005).

(9) P. S. Mohanty, R. Kesavamoorthy, K. Matsumoto, H. Matsuoka, and K. A. Venkatesan, Langmuir, 22, 4552 (2006).

(10) J. S. Downey, R. S. Frank, W. H. Li, and H. D. H. Stöver, Macromolecules, 32, 2838 (1999).

(11) J. S. Song and M. A. Winnik, Macromol., 38, 8300 (2005).

(12) K.C. Lee and S. Y. Lee, Macromol. Res., 15, 244 (2007). 
(13) J. Ugelstad, P. C. Mork, K. H. Kaggerud, T. Ellingsen, and A. Berg, Adv. Colloid Interf. Sci., 13, 101 (1980).

(14) K. Li and H. D. H. Stöver, J. Polym. Sci. Part A: Polym. Chem., 31, 3257 (1993).

(15) J. S. Downey, G. McIsaac, R. S. Frank, and H. D. H Stöver, Macromol., 34, 45 (2001).

(16) J. M. Lee, P. J. Saikia, K. Lee, and S. Choe, Macromolecules, 41, 2037 (2008).

(17) E. C. C. Goh and H. D. H. Stöver, Macromolecules, 35, 9983 (2002).

(18) W. H. Li and H. D. H. Stöver, J. Polym. Sci. Part A: Polym. Chem., 37, 2899 (1999).

(19) W. H. Li and H. D. H. Stöver, J. Polym. Sci. Part A: Polym. Chem., 37, 2295 (1999).

(20) R. S. Frank, J. S. Downey, and H. D. H. Stöver, J. Polym. Sci. Part A: Polym. Chem., 36, 2223 (1998).

(21) S. Yang, S. E. Shim, H. Lee, G. P. Kim, and S. Choe, Macromol. Res., 12, 519 (2004).

(22) S. E. Shim, S. Yang, H. Jung, and S. Choe, Macromol. Res., 12, 233 (2004).
(23) S. E. Shim, S. Yang, H. Jung, H. H. Choi, and S. Choe, J. Polym. Sci. Part A: Polym. Chem., 42, 835 (2004).

(24) J. M. Jin, S. H. Yang, S. T. Han, and S. Choe, J. Ind. Eng. Chem., 12, 268 (2006).

(25) J. M. Jin, J. M. Lee, M. H. Ha, K. Lee, and S. Choe, Polymer, 48, 3107 (2007).

(26) S. H. Han, K. K. Park, and S. H. Lee, Macromol. Res., 16, 120 (2008).

(27) S. E. Shim, S. Yang, J. M. Jin, Y. H. Chang, and S. Choe, Colloid Polym. Sci., 41, 283 (2004).

(28) D. D. Choi, K. Lee, and S. Choe, Inha Industry Partnership Institute Repub. Korea (2008).

(29) Z. Dai, X. Yang, and W. Huang, Polym. Int., 56, 224 (2007).

(30) M. S. Kim, S. K. Kim, and J. Y. Lee, Macromol. Res., 16, 178 (2008).

(31) M. Fernandez-Garcia, J. J. Martinez, and E. L. Madruga, Polymer, 39, 991 (1998).

(32) D. R. Hwang, J. Hong, and J. Lee, Macromol. Res., 16, 329 (2008). 